\title{
On Intrinsic Safety of Soft Robots
}

\author{
Haider Abidi and Matteo Cianchetti* \\ The BioRobotics Institute, Scuola Superiore Sant'Anna, Pisa, Italy
}

The rapidly growing field of soft robotics owes its success to the vast vistas of possibilities they promise. They may be utilized as standalone systems or work in harmony with the existing robotic technologies. Being based on soft and/or flexible materials, soft robots have usually high dexterity and, at the same time, they are also often considered "intrinsically safe." This is generally true and soft-bodied robots can be considered safer from a mechanical point of view, but this is sometimes improperly used. The identification of possible safety loopholes in soft robots is the subject of this paper. After a general overview of safety in robotics, we reported an overview of the main sources of unsafe conditions that may arise by the use of soft robotics technologies. Safety aspects are discussed in three categories: quasi-static, dynamic, and material failure. Some safety factors exclusive to soft robots such as whiplash-like effect and energy stored in highly strained elements are also introduced. Measures to avoid such unsafe conditions are

OPEN ACCESS

Edited by:

Rebecca Kramer,

Purdue University, USA

Reviewed by:

Stefano Palagi,

Max Planck Institute for Intelligent Systems (MPG), Germany Yigit Menguc,

Oregon State University, USA

*Correspondence: Matteo Cianchetti matteo.cianchetti@sssup.it

Specialty section: This article was submitted to Soft Robotics, a section of the journal Frontiers in Robotics and Al

Received: 17 October 2016

Accepted: 31 January 2017

Published: 20 February 2017

Citation:

Abidi H and Cianchetti M (2017) On Intrinsic Safety of Soft Robots.

Front. Robot. Al 4:5. doi: 10.3389/frobt.2017.00005 presented such as establishing operational limits and introduction of inspection regimes and arrest systems.

Keywords: soft robotics, smart materials, soft actuators, safety, physical human-robot interaction

\section{INTRODUCTION}

As robots become more and more sophisticated and ubiquitous, the question of safety becomes ever more important. Initially, humans and robots did not share work environments. Even so, the idea of human safety was essential, also becoming part of famous science fiction literature (Asimov, 1950). Today, with the advent of collaborative robotics, ensuring the safety of humans working nearby robots is critical. The highly active research field of physical human-robot interaction (pHRI) focuses on equipping robots with means of sensing humans within the work environment, managing collision strategies, and ensuring an accident-free collaborative environment through designs and control schemes (Shin et al., 2009; Haddadin, 2013; Sanan, 2013).

In order to evaluate the safety concerns related to robotics, certain metrics have been adopted over the years. De Santis et al. (2008) and Bicchi and Tonietti (2004) give an insight to the safety standards and injury evaluation criterions used in robotics. These criterions are not necessarily particular to robotics. They are also used in the automobile industry. The head injury criterion (HIC) is one of the most common criterions to measure the severity of harmful influences. Means of giving a quantitative value to different robots in terms of their danger potential have also been developed (Ikuta et al., 2003).

However, until recently, not many studies had been carried out to evaluate the possible injuries from rigid robots. Haddadin et al. (2009) conducted extensive tests on the subject, relating the tests to the HIC index. To avoid harmful interactions, the worst case scenarios of human-robot interactions were studied. 
Injuries may occur in two operation modes of the robot.

1. Injury in quasi-static operation mode: the robot motion is not fast and scenarios such as crushing could arise.

2. Injury in dynamic operation mode: the robot is in motion and effects such as transfer of inertia come to light.

To counter such events, new joint designs have been explored. Pioneering works by Bicchi and Tonietti (2004) indicated different means to reduce the inertia transfer by using compliant joints. In the early eras of robotics, compliance was considered as somewhat of a nuisance since it brought about many control challenges. Better models and control schemes and ubiquitous use of robots have made compliance important for safe robotics today (Albu-Schaffer et al., 2008).

Haddadin et al. (2009) indicated that playing with compliance has a limited effect. After a certain level, increasing joint compliance does not decrease the contact force (Van Damme et al., 2010). It was concluded that robots that are moving at less than $2 \mathrm{~m} / \mathrm{s}$ are safe for pHRI, independent of mass. The example of human walking straight into a wall is quoted as a simile. Speed limits and soft coverings are enough to ensure safety in case of unconstrained motion. In constrained motion, the use of torque sensing and other techniques such as visual based controls come into play, since robots may exert tremendous forces in a constrained environment. In this quest of searching for safe collaborative assistants, soft robots seem to appear as a Panacea that answers all concerns about safety. This paper prods at this notion of "intrinsic safety" and is focused on soft robots based on technologies made of elastomeric materials, namely flexible fluidic actuators (FFA), electroactive polymers (EAP), and cable-driven actuators. These are the most commonly used technologies, usually appearing as arm-like soft robots.

\section{SOFT ROBOTICS AND SAFETY}

The RoboSoft ${ }^{1}$ community [reported in Cianchetti et al. (2015)] defines soft robots as "soft robots/devices that can actively interact with the environment and can undergo "large" deformations relying on inherent or structural compliance," thus, including also the technologies covered in this paper. Born with the revolutionary aim of unhinging the basis of traditional robotics (Kim et al., 2013), soft-bodied robots (Wang and Iida, 2015) are now demonstrating how new abilities can be enabled, synergistically working with more traditional technologies based on hard materials (Laschi et al., 2016). They are strikingly different from the traditional rigid robots in terms of materials and mechanisms. They provide higher degrees of freedom and dexterity in narrow places (Shepherd et al., 2011; Cianchetti et al., 2014) and find applications in a variety of vistas from industrial to medical fields (Rus and Tolley, 2015). This, however, introduces difficulty in their control (Laschi and Cianchetti, 2014). They are lightweight and hence perfect for human collaborative environments like assistive robots (Cianchetti and Laschi, 2016). These qualities help develop

${ }^{1}$ RoboSoft is a Coordination Action on Soft Robotics funded by the European Commission. novel applications in industrial fields such as universal grippers (Brown et al., 2010) and enable the implementation of flexible chains. Soft robots also promise to solve the questions related to safety, partially, if not completely. This prospect of embodied safety has also accelerated the development of manipulators based on soft robotic technologies (Sanan et al., 2011). However, the authors argue that calling soft robots intrinsically safe, although generally true, carries some reservations.

Even soft plush toys for infants, made of innocuous soft materials, need to follow certain guidelines in order to be termed safe. ${ }^{2}$ The materials and mechanisms used for soft robots are wide and varied. A soft exterior may be sufficient to ensure safety by reducing the mechanical impulse transferred; this of course depends on other parameters such as the reflected inertia of the robot and the rate of change of velocity during the impact. One may argue that given the relatively low masses and velocities of soft robots, this could be a validation of the intrinsic safety, in the case of blunt impacts at least. However, due to the nature of soft robots, low velocities can give birth to hazardous situations. Moreover, some other safety concerns may also be present. They are discussed below under material failure, static, and dynamic operational modes.

\section{MATERIAL FAILURE}

Unlike rigid robotics, where the construction material is considerably robust and is less likely to fail, material failure is a significant safety concern in soft robots. Abrasion becomes a serious threat, intensified by tearing possibilities. Soft materials have poor tear resistance, especially when large deflections come into play.

Material failure can be subdivided based on the actuation technology used. The most obvious safety hazard for FFAs is a sudden release of pressure. This may be due to material failure or rupture due to external influences. Pneumatic actuators often tend to have a ballooning effect, which is fundamental for actuation. Being enveloped in a covering, the ballooning is usually not directly exposed to the workspace (Cianchetti et al., 2013), but it may not always be possible to shield the ballooning area from the work environment. If in direct contact with humans, a tear could cause sudden release of the elastic energy stored in the material, thrusting it to a nearby object. Such cases are important for close contact robots such as used in surgical and assistive fields. Although not common, material failures do occur in medical procedures [e.g., in Katayama et al. (2010)]. Moreover, the likelihood of them happening during use increases due to effects such as aging, which causes structural changes leading to increased stiffness in elastomeric materials. Environmental conditions such as temperature and chemicals have an influence on the rate of aging (Leslie et al., 2008). Another effect, which makes elastomers' mechanics even more complex, is the Mullins effect (Mullins, 1948) that leads to strain softening upon first expansion. This is due to the breaking of polymeric chains at microlevel, which implies a permanent change in the elastomeric

${ }^{2}$ https://www.bsigroup.com/en-GB/about-bsi/media-centre/press-releases/ 1999/7/New-Toy-Standard-to-Improve-Safety-of-Toys/. 
network. These factors highlight the need of rigorous considerations to concepts like "time of use" and "unexpected strains."

Ruptures and leakages may expose the human to high pressures. Normally operating pressures of pneumatic actuators are in the range of 2 bar, but it may go as high as $8-10$ bar. Pressures in these ranges may also be dangerous. As an example, 8 bar pressure applies a force of $0.157 \mathrm{~N}$ through a hole of $0.5 \mathrm{~mm}$ diameter. This is not significant enough to cause a fracture or deep tissue damage, however, for sensitive soft tissues, such as the eye, it can cause damage. Waller et al. (1993) shows a pressure of 0.02 bar using a blunt edge needle (23 gauge) being enough to cause penetration. This of course is an extreme example, but it shows that certain safety measures need to be taken. On the other hand, if undetected, leakages may lead to unwanted pressurization of the environment. This is another point of concern in medical robotics. Though surgical interventions using laparoscopes involve insufflation, the pressure is carefully regulated. Increasing insufflation pressure can lead to adverse effects like cardiac arrhythmia and respiratory complications (Perrin and Fletcher, 2004).

In light of the above discussion, the design of soft robots should be based on the material properties such as toughness and abrasion resistance (also considering aging effects). Operational limits should be applied such that localized stresses near rupture stress are not reached. At the same time, care should be taken in the fabrication so as to avoid failure sites and also during use to avoid failure due material wear and tear. A flexible covering such as a braided sheath may protect the soft manipulator from possible causes of rupture, while a pressure arrest system can be used to help mitigate the effects of a rupture.

For cable-based designs, snapping can be a source of hazard. A snap of cables or the structure itself can release elastic energy stored in the stretched manipulator. If the cables are composed of metallic materials, they may be even more dangerous, causing parts of the soft robot to fling rapidly. The snapping of a stretched rubber band is similar example. Limiting the maximum cable actuation force can prevent this concern.

Some soft robotics technologies require electrical and thermal insulation, such as those based on EAPs. If not properly designed, a failure of the dielectric material due to the reaching of breakdown voltage levels could result in high current flow. However, these are general concerns that are not specific to soft robots only.

\section{DYNAMIC MODE}

The severity of impact can be calculated from impulse. Hence, the critical factors are force and the time it acts for. The force of impact can be calculated by various means. If considering a soft robot as a beam, the relation between deflection, $y$, and force, $F$, is given by

$$
F=\frac{\partial^{2}}{\partial x^{2}}\left(E I \frac{\partial^{2} y}{\partial x^{2}}\right)+m \frac{\partial^{2} y}{\partial t^{2}}
$$

where $E$ is Young's modulus, $I$ is the moment of inertia, $x$ parameterizes the beam length, $t$ represents time, and $m$ is the mass per unit length. It is clear that having a low stiffness (in terms of elastic modulus) would lead to lower forces (Timoshenko, 1941). The premise of safety in soft robots is based on this low stiffness. Compared to rigid robots, the modulus of elasticity of "soft" materials is usually reduced by a factor of 10 (Rus and Tolley, 2015), even though methods of varying stiffness is an active area of research (a recent review paper covers this topic - Manti et al. 2016). Despite of this softness, high velocities will still be dangerous due to the effect of mass, as discussed in the introduction pertaining to rigid robots.

Another method of measuring impact forces is by measuring the rate of change of momentum, making mass and velocities the critical factors. The more "soft" a robot is, the greater it falls in the inelastic domain of collisions. The principle of conservation of momentum or the use of coefficient of restitution (ratio of the relative velocities before and after collision) can be used to compute post impact velocities.

Force models for impact analysis (like the Hunt-Crossley model) do not rely on beam equations. They use the rate of deformation for calculating impact forces (Gilardi and Sharf, 2002) and represent them as

$$
F_{\text {impact }}=F_{\dot{\delta}}(\dot{\delta})+F_{\delta}(\delta)
$$

where $F$ represents force of impact, $\delta$ and $\dot{\delta}$ represent indentation and the rate of indentation, respectively. The terms of velocities and material properties are implicitly used in these models. Mazzocchi et al. (2015) presents an example of soft interface design.

Thus, the factors defining the severity of impact are the mass, velocity, and material properties (such as "softness") of the robot. No surprises there. Low velocities are good practice to avoid safety issues in dynamic motion, but in soft robotics, even limited velocities can give rise to high impact scenarios. This is demonstrated by a so-called whiplash-like effect. Cephalopods arms and tentacles or elephant trunks often provide inspiration for dexterity. The end of these arm-like manipulators are equipped with appendages such as grippers or they may be carrying a payload. The arms usually move slowly, however, the velocity of the end effector may increase considerably if the arm encounters an obstacle in the workspace. Figure 1 represents the

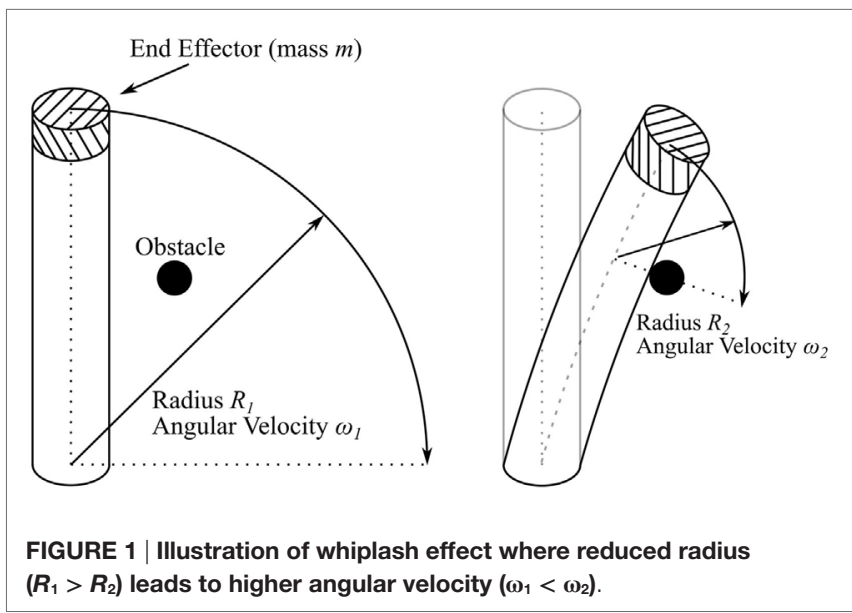


idea. Suppose a manipulator is moving with a tip angular velocity of $\omega$, carrying a mass, $m$, at the tip. Let the initial radius of the rotation be represented by $R$. If the manipulator is rigid, the tip speed will remain the same before and after the collision with an obstacle. However, if the manipulator is compliant, the radius of rotation will change. This is due to the fact that a sufficiently low flexural rigidity (term $E I$ of the beam model above) translates to large deflections even at low forces. An extreme example of this is a mass revolving about a point held in orbit by a string. The new radius will stem from the point of collision ending at the tip of the manipulator. The law of conservation of momentum dictates an increase in speed of the tip as the manipulator tries to wind around the obstacle.

Angular momentum is represented by the equation

$$
M=I \times \omega
$$

where $M$ is the momentum, $I$ is the moment of inertia.

$$
I=m R^{2}
$$

If the radius is reduced, the effect is squared in the moment of inertia, hence the angular velocity will increase in order to keep the angular momentum constant. Since the velocity is represented by the expression

$$
v=R \times \omega
$$

If the radius of rotation is scaled down to $R / a$ ( $a$ being the reduction factor) the overall velocity would increase by the same factor. This increased velocity in turn can result in a strong impact. It should be noted that the mass in question is the tip mass and not the mass of the robot arm itself. At the same time, in most cases, the increase of velocity will also depend on the flexural rigidity (unlike a string, high rigidity due to material or geometric reasons will resist the formation of a pivot point, especially at small radii). However, whiplash is not very likely in confined regions such as in the case of surgical scenarios. There is not enough volume to attain high speeds, but in the case of assistive and industrial robots, it is worthwhile to incorporate means of control so the term $a v$ is below a safe limit.

\section{DISCUSSION}

There are undeniable advantages in the use of soft robots, but the claim of intrinsic safety could still be misleading. While rigid robots can exert very large crushing forces at singularities where joint velocities tend to infinity, in soft robotics, such scenarios are improbable. Though mathematical singularities may arise due to modeling assumptions, mechanical singularities are not present; thanks to the continuum nature of soft “joints." Moreover, large deformations cause an increase in the contact area, reducing the crushing effect, if present. At the same time, viscous components of elastomers cause energy losses, leading to a reduction in the transferred force. However, hazards due to material failure and whiplash effects can be present. In light of the above discussion, the following measure can be taken to avoid possible unsafe scenarios:

1. Mathematical modeling, material tests, and finite element analysis can be used to identify the rupture strength and set a suitable safety limit of stress on powering;

2. The use of highly deformable sheaths (like in FFA) protects the manipulators from possible causes of rupture and helps contain the effects of failure;

3. Special care should be taken in the fabrication process to avoid introduction of potential material failure sites such as bubbles;

4. Given the low tear resistance of elastomers, inspections for cuts and leakages are essential before use and during maintenance;

5. A pressure arrest system for pneumatic actuators is advisable to prevent sudden pressure release scenarios;

6. Aging causes a more fragile mechanical behavior, thus an estimation of the device life time has to be taken into consideration in the specific material choice;

\begin{tabular}{|c|c|c|c|}
\hline Hazard & Statics - material failure related hazards & \multicolumn{2}{|c|}{ Dynamics - velocity related hazards } \\
\hline Technology & Release of actuating medium & Release of elastic energy & Whiplash \\
\hline FFA & $\begin{array}{l}\text { - Limit actuation pressure to remain within safe stress limits } \\
\text { - Prevent bubbles during fabrication to avoid fabrication defects } \\
\text { - Define life and conduct regular inspections to avoid age related } \\
\text { failures } \\
\text { - Use flexible covering sheaths to protect against sharp objects } \\
\text { - Install pressure arrest systems to prevent environmental } \\
\text { pressurization }\end{array}$ & \multirow{2}{*}{$\begin{array}{l}\text { - Avoid possibility of slippage while actuating } \\
\text { - Limit actuation pressure to avoid excessive } \\
\text { energy storage }\end{array}$} & \multirow{3}{*}{$\begin{array}{l}\text { - Limit actuation velocity } \\
\text { - Use of proximity sensors } \\
\text { to identify obstacles }\end{array}$} \\
\hline EAP & $\begin{array}{l}\text { - Limit actuation voltage to prevent reaching breakdown voltages } \\
\text { - Use appropriate electrical insulation covering } \\
\text { - Define useful life to avoid age related failures }\end{array}$ & & \\
\hline $\begin{array}{l}\text { Cable and } \\
\text { tendon }\end{array}$ & - & $\begin{array}{l}\text { - Ensure proper cable anchoring } \\
\text { - } \text { Avoid possibility of slippage while actuating } \\
\text { - Limit actuation force to avoid excessive } \\
\text { energy storage }\end{array}$ & \\
\hline
\end{tabular}

TABLE 1 | Table of hazards and their preventive measures associated with flexible fluidic actuators (FFA), cable actuation, and electroactive polymers (EAP) technologies. 
7. Mullins effect can change the material properties of the elastomers, leading to a decreased stiffness, which is not directly connected to a cause of failure, but could lead to the use of higher than required, and hence dangerous, pressures;

8. In order to counter the whiplash effect, a velocity limit can be implemented based on the flexural rigidity of the robot to prevent a pivot point being formed at the impact site;

9. Pressure and force limiters can be installed in the control of the robots to avoid material failure and the buildup of elastic energy (as is usually done in rigid robots).

Table 1 summarizes the hazards in soft robots and lists the possible safety measures for each case.

\section{CONCLUSION}

Similar to rigid robots, forces in the quasi-static and dynamic regime need to be considered in soft robots and, in addition, material failure scenarios need to be addressed too. However, while

\section{REFERENCES}

Albu-Schaffer, A., Eiberger, O., Grebenstein, M., Haddadin, S., Ott, C., Wimbock, T., et al. (2008). Soft robotics. IEEE Robot. Autom. Mag. 15, 20-30. doi:10.1109/ MRA.2008.927979

Asimov, I. (1950). I, Robot. Greenwich, CT: Fawcett Publications.

Bicchi, A., and Tonietti, G. (2004). Fast and "soft-arm" tactics [robot arm design]. IEEE Robot. Autom. Mag. 11, 22-33. doi:10.1109/MRA.2004.1310939

Brown, E., Rodenberg, N., Amend, J., Mozeika, A., Steltz, E., Zakin, M. R., et al. (2010). Universal robotic gripper based on the jamming of granular material. Proc. Natl. Acad. Sci. U.S.A. 107, 18809-18814. doi:10.1073/pnas.1003250107

Cianchetti, M., Calisti, M., Margheri, L., Kuba, M., and Laschi, C. (2015). Bioinspired locomotion and grasping in water: the soft eight-arm OCTOPUS robot. Bioinspir. Biomim. 10, 035003. doi:10.1088/1748-3190/10/3/035003

Cianchetti, M., and Laschi, C. (2016). Pleasant to the touch. IEEE Pulse Mag. 3, 34-37. doi:10.1109/MPUL.2016.2539799

Cianchetti, M., Ranzani, T., Gerboni, G., De Falco, I., Laschi, C., and Menciassi, A. (2013). "STIFF-FLOP surgical manipulator: mechanical design and experimental characterization of the single module," in Proceedings of International Conference on Intelligent Robots and Systems (IROS) (Tokyo), 3576-3581.

Cianchetti, M., Ranzani, T., Gerboni, G., Nanayakkara, T., Althoefer, K., Dasgupta, P., et al. (2014). Soft robotics technologies to address shortcomings in today's minimally invasive surgery: the STIFF-FLOP approach. Soft Robot. 1, 122-131. doi:10.1089/soro.2014.0001

De Santis, A., Siciliano, B., De Luca, A., and Bicchi, A. (2008). An atlas of physical human-robot interaction. Mech. Mach. Theory 43, 253-270. doi:10.1016/j. mechmachtheory.2007.03.003

Gilardi, G., and Sharf, I. (2002). Literature survey of contact dynamics modelling. Mech. Mach. Theory 37, 1213-1239. doi:10.1016/S0094-114X(02) 00045-9

Haddadin, S. (2013). Towards Safe Robots: Approaching Asimov's 1st Law, Vol. 90. Berlin, Heidelberg: Springer-Verlag.

Haddadin, S., Albu-Schäffer, A., and Hirzinger, G. (2009). Requirements for safe robots: measurements, analysis and new insights. Int. J. Robot. Res. 28, 1507-1527. doi:10.1177/0278364909343970

Ikuta, K., Ishii, H., and Nokata, M. (2003). Safety evaluation method of design and control for human-care robots. Int. J. Robot. Res. 22, 281-297. doi:10.1177/02 78364903022005001

Katayama, T., Sakoda, N., Yamamato, F., Ishizaki, M., and Iwasaki, Y. (2010). Balloon rupture during coronary angioplasty causing dissection and intramural hematoma of the coronary artery; a case report. J. Cardiol. Cases 1, e17-e20. doi:10.1016/j.jccase.2009.06.002 rigid robots may apply crushing forces due to large magnitude, soft robots are unlikely to have such an influence owing to their compliant nature and low mass. The main conclusion that can be drawn from this analysis is that though limited in comparison to traditional rigid robotics, soft robots may suffer from some safety concerns. These have been reported in this paper together with some guidelines useful to face and limit them so that the complete potential of soft robotics can be exploited.

\section{AUTHOR CONTRIBUTIONS}

All authors listed have made substantial, direct, and intellectual contribution to the work, and approved it for publication.

\section{ACKNOWLEDGMENTS}

The authors acknowledge the support of the European Commission through the RoboSoft CA (project no. 619319, ICT FET-Open).

Kim, S., Laschi, C., and Trimmer, B. (2013). Soft robotics: a bioinspired evolution in robotics. Trends Biotechnol. 31, 287-294. doi:10.1016/j.tibtech.2013.03.002

Laschi, C., and Cianchetti, M. (2014). Soft Robotics: new perspectives for robot bodyware and control. Front. Bioeng. Biotechnol. 2:3. doi:10.3389/ fbioe.2014.00003

Laschi, C., Mazzolai, B., and Cianchetti, M. (2016). Soft robotics: technologies and systems pushing the boundaries of robot abilities. Sci. Robot. 1, eaah3690. doi:10.1126/scirobotics.aah3690

Leslie, L. J., Jenkins, M. J., Shepherd, D. E. T., and Kukureka, S. N. (2008). The effect of the environment on the mechanical properties of medical grade silicones. J. Biomed. Mater. Res. 86B, 460-465. doi:10.1002/jbm.b.31042

Manti, M., Cacucciolo, V., and Cianchetti, M. (2016). Stiffening in soft robotics: a review of the state of the art. IEEE Robot. Autom. Mag. 23, 93-106. doi:10.1109/ MRA.2016.2582718

Mazzocchi, T., Diodato, A., Ciuti, G., Micheli, D. M. D., and Menciassi, A. (2015). "Smart sensorized polymeric skin for safe robot collision and environmental interaction," in International Conference on Intelligent Robots and Systems (IROS) (Hamburg: IEEE/RSJ), 837-843.

Mullins, L. (1948). Effect of stretching on the properties of rubber. Rubber Chem. Technol. 21, 281-300. doi:10.5254/1.3546914

Perrin, M., and Fletcher, A. (2004). Laparoscopic abdominal surgery. Contin. Educ. Anaesth. Crit. Care Pain 4, 107-110. doi:10.1093/bjaceaccp/mkh032

Rus, D., and Tolley, M. T. (2015). Design, fabrication and control of soft robots. Nature 521, 467-475. doi:10.1038/nature14543

Sanan, S. (2013). Soft Inflatable Robots for Safe Physical Human Interaction [Doctoral Dissertation]. Carnegie Mellon University, Pittsburgh, PA.

Sanan, S., Moidel, J., and Atkeson, C. G. (2011). "A continuum approach to safe robots for physical human interaction," in International Symposium on Quality of Life Technology, Toronto.

Shepherd, R. F., Ilievski, F., Choi, W., Morin, S. A., Stokes, A. A., Mazzeo, A. D., et al. (2011). Multigait soft robot. Proc. Natl. Acad. Sci. U.S.A. 108, 20400-20403. doi:10.1073/pnas.1116564108

Shin, D., Sardellitti, I., Park, Y. L., Khatib, O., and Cutkosky, M. (2009). Design and control of a bio-inspired human-friendly robot. Int. J. Robot. Res. 29, 571-584. doi:10.1177/0278364909353956

Timoshenko, S. (1941). Strength of Materials. New York: D. Van Nostrand Company.

Van Damme, M., Beyl, P., Vanderborght, B., Van Ham, R., Vanderniepen, I., Matthys, A., et al. (2010). "The role of compliance in robot safety," in Proceedings of the Seventh IARP Workshop on Technical Challenges for Dependable Robots in Human Environments (DRHE) (Toulouse), 65-71.

Waller, S. G., Taboada, J., and O'Connor, P. (1993). Retrobulbar anesthesia risk. Ophthalmology 100, 506-510. 
Wang, L., and Iida, F. (2015). Deformation in soft-matter robotics: a categorization and quantitative characterization. IEEE Robot. Autom. Mag. 22, 125-139. doi:10.1109/MRA.2015.2448277

Conflict of Interest Statement: The authors declare that the research was conducted in the absence of any commercial or financial relationships that could be construed as a potential conflict of interest.
Copyright (c) 2017 Abidi and Cianchetti. This is an open-access article distributed under the terms of the Creative Commons Attribution License (CC BY). The use, distribution or reproduction in other forums is permitted, provided the original author(s) or licensor are credited and that the original publication in this journal is cited, in accordance with accepted academic practice. No use, distribution or reproduction is permitted which does not comply with these terms 